\title{
Spinal Cord Stimulation for Control of Pain
}

\author{
Stephen Falowski MD \\ Thomas Jefferson University
}

Follow this and additional works at: https://jdc.jefferson.edu/jhnj

Part of the Neurology Commons

Let us know how access to this document benefits you

\section{Recommended Citation}

Falowski MD, Stephen (2008) "Spinal Cord Stimulation for Control of Pain," JHN Journal: Vol. 4 : Iss. 1 , Article 5.

DOI: https://doi.org/10.29046/JHNJ.004.1.002

Available at: https://jdc.jefferson.edu/jhnj/vol4/iss1/5

This Article is brought to you for free and open access by the Jefferson Digital Commons. The Jefferson Digital Commons is a service of Thomas Jefferson University's Center for Teaching and Learning (CTL). The Commons is a showcase for Jefferson books and journals, peer-reviewed scholarly publications, unique historical collections from the University archives, and teaching tools. The Jefferson Digital Commons allows researchers and interested readers anywhere in the world to learn about and keep up to date with Jefferson scholarship. This article has been accepted for inclusion in JHN Journal by an authorized administrator of the Jefferson Digital Commons. For more information, please contact: JeffersonDigitalCommons@jefferson.edu. 


\section{Spinal Cord Stimulation for Control of Pain}

\section{Stephen Falowski, MD}

Department of Neurological Surgery, Thomas Jefferson University, Philadelphia, Pennsylvania

\section{Introduction}

Spinal cord stimulation (SCS) is an adjustable, non-destructive procedure which delivers therapeutic doses of electrical current to the spinal cord for the management of neuropathic pain. The most common indications include post-laminectomy ("failed back surgery") syndrome, complex regional pain syndrome (CRPS), ischemic limb pain, and angina. Other reported applications include visceral/abdominal pain, cervical neuritis pain, spinal cord injury pain, post-herpetic neuralgia, and neurogenic thoracic outlet syndrome.

\section{History}

Advances in technology have driven the popularity of the field. Initially, the contact combinations could only be hardwired, and could not be reprogrammed after the pulse generator was implanted. In the beginning, only radio-frequency (RF) driven passive receivers were available. In the midseventies, the first implantable pulse generator powered by a lithium battery was introduced into the market. In 1980, the first percutaneous electrode was produced, and this could be reprogrammed non-invasively through an external transmitter. ${ }^{1}$

\section{Mechanisms of Action}

SCS began when Melzack and Wall ${ }^{2}$ noted that stimulation of large peripheral nerve fibers could block the sensation of pain. In 1967, Shealy ${ }^{3}$ inserted the first dorsal column stimulator in a human suffering from terminal metastatic cancer. Later approaches implanted electrodes via a laminectomy in the subarachnoid space, between the two layers of the dura, or in the epidural space. ${ }^{4-6}$ Subsequently, less invasive percutaneous techniques were introduced. ${ }^{\text {? }}$

The exact mechanisms of action of SCS remain unclear, although computer modeling work has shed some light on the distribution of complex electrical fields within the spine. ${ }^{8-13}$ Animal studies suggest that the SCS triggers the release of serotonin, substance $\mathrm{P}$, and GABA within the dorsal horn. ${ }^{14,15}$ It is not known whether stimulation of different sites-peripheral nerves, dorsal columns, or supra-lemniscal pathways-will trigger equivalent mechanisms of action.

\section{Indications}

SCS has been successfully used for a variety of pain conditions (Table I). Experience suggests that, in selected patients, SCS can produce at least $50 \%$ pain relief in $50-60 \%$ of the implanted patients. Interestingly, with the proper follow-up care, these results can be maintained over several years.

\section{Complex Regional Pain Syndrome (CRPS)}

The implementation of SCS in individuals with CRPS type I is more difficult than with any other patients groups. There is risk of aggravating the original pain or causing a new pain at the implanted hardware site is greater than with any other diagnostic category mentioned. The CPRS-affected areas may be too widespread for effective stimulation.

In 1989, Barolat et al. reported reduction of pain in ten out of thirteen patients implanted. No patients in that series were made pain free but all ten reported a definite difference from stimulation. ${ }^{16}$ In 1997 , Kumar et al. reported on twelve patients with permanently implanted leads ${ }^{17}$ : eight patients enjoyed near complete resolution of their symptoms and four also maintained good relief.

Kemler et al. reported 23 additional cases with $78 \%$ of the patients reporting improvement. ${ }^{18} \mathrm{~A}$ later study ${ }^{19}$ compared patients randomized to SCS to those treated with physical therapy. In the SCS group, $67 \%$ of patients experienced significant pain relief which persisted at 6 months. However, no functional improvement was observed in either group. A 5-year follow-up on the SCS group indicated that the effects of SCS diminished over time for these patients. ${ }^{20}$
Oakley and Weiner reported a prospective study of 19 patients with CRPS implanted with spinal cord stimulation systems. ${ }^{21}$ Of the ten patients in whom detailed long-term efficacy data was available, three reported full relief from their pain and seven, partial beneficial relief.

Three additional prospective studies without matched controls have been reported. ${ }^{21-23}$ Two of the studies reported success rates with an $84 \%$ overall success rate. The third study by Calvillo et al. ${ }^{22}$ reported a significant improvement in pain scores and a $>50 \%$ reduction in narcotic use by $44 \%$ of subjects. In eight retrospective studies the overall success rate was $84 \% 192$ patients. ${ }^{24}$

\section{Post-laminectomy syndrome aka Failed back surgery syndrome (FBSS)}

Post-laminectomy syndrome is a broad term which can include pain localized to the lower lumbar region or the buttocks, persistent radicular pain, or diffuse lower extremity pain. Most published series distinguish between back and leg pain, but seldom define the details of the pain syndromes. SCS is accepted in the treatment of leg pain, but its widespread use for relief of pain in the lower lumbar area still remains to be defined.

A technical challenge has been to provide stimulation in the low back, where paresthesia is often replaced over time by an unpleasant segmental band of stimulation from the thoracic roots. Previous pioneering work by Jay $\operatorname{Law}^{25,26}$ has shown that stimulation in the low back can be obtained only if one uses multiple arrays of closely spaced bipolar electrodes at T9-T10. North et al. have shown that one single quadripolar electrode in midline has the ability to stimulate the axial low back. ${ }^{27}$ Flanking the cathode by lateral anodes also appears to increase the discomfort threshold theoretically. ${ }^{28}$

Marchand et al. ${ }^{29}$ conducted a prospective randomized controlled study examining patients with at least one prior surgery for chronic back pain secondary to trauma. Each patient used a SCS and acted as his or her own control. 
Although a small trial, with only eight patients, pain scores were significantly reduced with SCS compared to placebo stimulation.

The longitudinal studies by North showed that in patients with post-surgical lumbar arachnoid or epidural fibrosis without surgically remediable lesions, SCS provided at least $50 \%$ pain relief in $53 \%$ of patients at 2.2 years. ${ }^{30}$ North et al. also conducted a study randomizing patients with FBSS to either repeat back surgery or SCS surgery, ${ }^{33}$ allowing crossover after six months. Ten of fifteen patients crossed over from back surgery to SCS, while only two of twelve patients crossed over from SCS to back surgery.

Turner et al. ${ }^{31}$ systematically reviewed a total of 41 articles from 1966 to 1994 and noted that approximately $50-60 \%$ of patients with post-laminectomy greater than $50 \%$ pain relief was attained from the use of SCS. In 1996, Burchiel et al. conducted a prospective multicenter study with one year follow-up and also reported $55 \%$ successful stimulation. ${ }^{32}$

\section{Angina}

There are well documented reports in the literature revealing uniformly good results using SCS to relieve anginal pain. ${ }^{34-38}$ Further, the results have been maintained in long term follow-up and have been substantiated by a reduction in the intake of nitrates as well. Interestingly, other findings have supported the evidence that SCS has effects that go beyond pain relief. The observations that there is less ST segment depression and that the exercise capacity, the time to angina and the recovery time all improve with stimulation may suggest that there is a reduction in ischemia. In a positron emission tomography study, a redistribution of myocardial flow in favor of ischemic parts of the myocardium has been demonstrated as a long term effect of spinal cord stimulation, both at rest and after pharmacologic stress induction. $^{39}$

Vulink et al. conducted a prospective study on quality of life changes in patients with refractory angina pectoris implanted with SCS. They found that both the pain and the health aspects of quality of life improved significantly after 3 months of SCS. Further, social, mental and physical aspects of quality of life were found improved after one year of SCS. ${ }^{40}$

Hautvast et al. ${ }^{41}$ implanted SCS in patients with stable angina pectoris and randomized them. One group's remained inactivated while the other group was instructed to use the stimulator three times per day for one hour and with any angina attack. At 6 weeks, compared with controls, the treatment group had increased exercise duration and time to angina, and decreased anginal attacks and sublingual nitrate consumption. Also, observed was a decrease in ischemic episodes on EKG, as well as a decrease in observed ST segment depressions on exercise EKG. There was an increase in perceived quality of life and decrease in pain.

Mannheimer et al. $^{42}$ randomized 104 patients accepted for CABG to receive either CABG $(n=51)$ or SCS $(n=53)$ in the ESBY study. This study demonstrated that patients randomized to SCS showed a greater than $30 \%$ improvement in Nottingham Health Profile (NHP) scores compared with baseline, which was significant and comparable to the improvement shown by patients randomized to $\mathrm{CABG}^{43}$ These results were consistent on follow up after four years. It is important to know that the five-year mortality of $27.9 \%$ in the ESBY study was similar between those receiving SCS and those who received CABG, with no difference in the percentage of cardiac deaths. The ESBY study showed that cardiac events were similar across the groups, but that there was significantly more cerebrovascular events observed in the CABG group.

Both groups experienced a significant reduction in both the number of angina attacks and the consumption of nitrates. There was no significant intergroup difference regarding these parameters. In another prospective study of 104 patients who underwent SCS implantation for refractory angina pectoris there was a significant decrease in angina episodes at rest, angina episodes with activity, and total angina episodes ${ }^{44}$

DeJongste et $\mathrm{al}^{36}$ randomized seventeen patients with angina to an active treatment group (i.e. SCS implantation) and a control group. The control group was followed for two months followed by SCS implantation. Both groups were followed for a total of 12 months. This study also revealed a statistically significant reduction in the incidence of angina attacks and in the consumption of nitrates.

Five additional studies are reported to be prospective but without matched controls. ${ }^{36,40,45-48}$ Each of these revealed significant benefit from spinal cord stimulation. The benefit indices ranged from reduction in angina attacks, decrease nitrate consumption, decrease in NYHA grade and improvement in NHP grade.

Andersen et al. reported that out of 45 patients treated with SCS for anginal pain, there were three who had also survived a myocardial infarction $^{49}$ : all three patients noticed the pain to be different and unrelieved with SCS and all patients correctly guessed that the pain was due to a myocardial infarction. The authors concluded that SCS reduces the severity of anginal attack, but was unable to suppress the conduction and perception of cardiac pain signals which act as alarm signals of cardiac distress. Similarly, Murray et al. have shown that SCS for refractory angina is effective in preventing hospital admissions without masking the ischemic symptoms or leading to silent infarction. ${ }^{50}$

How SCS reduces angina is unclear. Hautvast et al. ${ }^{41}$ demonstrated no significant changes in heart rate variability after 6 wks, concluding that autonomic modulation of heart rate may not be the mechanism of action. There SCS may reduces myocardial ischemia via homogenization of myocardial blood perfusion. ${ }^{39,51}$ SCS can improve lactate metabolism in the heart muscle, and lactate metabolism, oxygen demand and blood flow in the coronary sinus. ${ }^{52}$ SCS does not effect variability in heart rates or cardiac arrhythmias. ${ }^{35,53,54}$

We do not know whether the pain relief is due to direct depression of the nociceptive signals in the spinal cord or whether there is secondary gain from a reduction in the ischemia. $^{55,56}$ Foreman has shown that dorsal column stimulation inhibits the activity of spinothalamic tracts cells evoked by activation of the cardiac sympathetic afferents or by intracardiac bradykinin. ${ }^{14}$ On the other hand the stimulation might producing a prolonged inhibition of the hyperactive sympathetic system, as was shown experimentally in the rat by Linderoth et al. ${ }^{15}$ The most appropriate electrode location for the treatment of angina pectoris is most likely the lower cervical and upper thoracic region, although some have reported successful higher cervical placements. ${ }^{42}$ Another consideration is continuous versus cyclical use of SCS. In practice, patients using SCS for angina pectoris often use a low intensity stimulation for several hours per day for prophylactic purposes. ${ }^{57}$ Recently, a randomized control study demonstrated improvement in functional status and symptoms in treatment arms with conventional or sub threshold stimulation in comparison to a low output placebo treatment arm. ${ }^{58}$

\section{Abdominal/Visceral Pain Syndromes}

Approximately $20 \%$ of the population in United States have abdominal pain. There are many etiologies for abdominal pain including 
gastrointestinal, genitourinary, musuloskeletal and nervous systems. Treatment modalities have included cognitive- behavioral, physical, and pharmacological therapies. Other more invasive therapies have extended to include celiac plexus blocks and celiac ganglia destruction. Some studies have demonstrated some localization in the spinal cord for visceral pain secondary to malignancy. Midline myelotomy through the dorsal columns at the level of T10 has shown success in eight patients with refractory pelvic cancer pain. This was also demonstrated in animal studies where dorsal column activity was observed in pelvic visceral nociception. ${ }^{59}$

Several studies have demonstrated the benefit of SCS in abdominal visceral disease. Ceballos et al..$^{60}$ demonstrated reduction in pain scores and decrease in narcotic use in a patient treated for mesenteric ischemia. Krames et al. ${ }^{61}$ described a patient treated for irritable bowel syndrome who was developing escalating pain and diarrhea. In the first 6 months there was a subjective decrease in pain on a 10-point scale from $9 / 10$ to $2 / 10$, with only two diarrhea episodes and with significant reduction in pain medications. There was some return of pain after ten month follow-up, but still a significant reduction in diarrhea. Khan et al. ${ }^{62}$ reported on the largest series with nine patients with refractory abdominal pain, all of whom had a significant improvement in pain scores as well as decreased narcotic use at six to eight month follow up.

Tiede et al. described treatment of refractory abdominal pain in two patients. Both patients had a significant history including multiple abdominal surgeries and failed conservative measures. Each patient had an element of postprandial abdominal pain with associated nausea and vomiting. In both patients the leads were placed at the T2 level with significant improvement in pain, decreased narcotic use and increase functioning, such as return to work $^{63}$. Kapur et al. ${ }^{64}$ recently described relief of abdominal pain associated with colchicine intolerant or resistant patients with familial Mediterranean fever by placement of the electrodes at the lower thoracic levels.

More recent studies have looked at the treatment of visceral pelvic pain with reference to the dorsal columns and spinal cord stimulation. Kapural et al. ${ }^{65}$ reported on the value of neurostimulation for chronic visceral pelvic pain in six female patients with the diagnosis of long-standing pelvic pain. These patients had a history of endometriosis, multiple surgical explorations, and dyspareunia. At an average follow up of 30 months there was a significant decrease in the pain score with an average of more than $50 \%$ pain relief, with a decrease in opiate use.

Visceral innervation follows the embryologic origin and location of the viscera and is arranged in viscerotomes, analogous to cutaneous dermatomes. ${ }^{66}$ The viscera obtain their innervation via the sympathetic and parasympathetic pathways. The parasympathetics carry their afferents to anterior and posterior vagal trunks and are therefore not as amendable to spinal cord stimulation. The sympathetics carry nociceptive information from the viscera to spinal nerve roots making them a more viable target. The sympathetic afferents in the lower six thoracic and the upper three lumbar spinal segments have been shown to transmit painful impulses from the viscera ${ }^{67}$.

\section{Equipment (Electrodes and pulse generators)}

There are various implantable technologies existing for SCS. These include trial percutaneous electrodes, permanent percutaneous

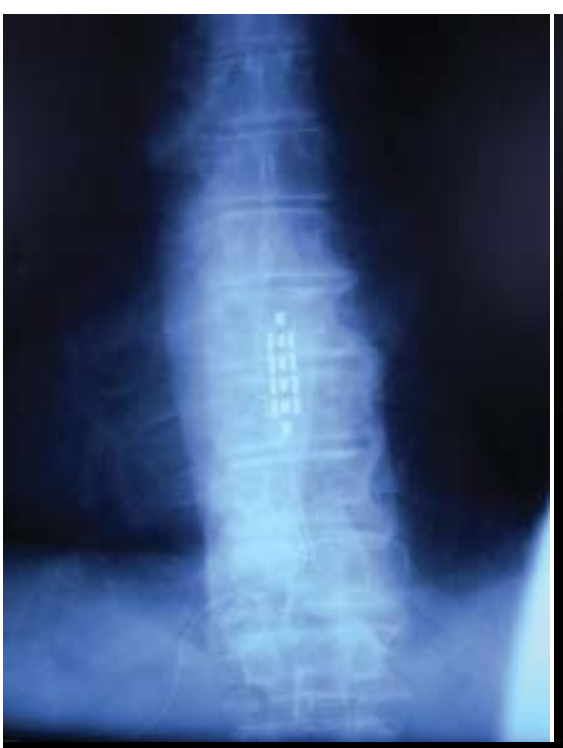

electrodes, permanent plate electrodes, totally implantable rechargeable and non-rechargeable pulse generators (IPG) and radiofrequency (RF) driven pulse generators.

\section{Percutaneous electrodes}

Percutaneous electrodes can be inserted without much dissection and can easily be removed in the implanting physician's office. During implantation, these electrodes can be advanced over several segments in the epidural space, allowing testing of several spinal cord levels to assess for optimal electrode position.

Contemporary percutaneous electrodes are slim electrodes, only a few millimeters in diameter and containing four or eight contacts (referred to as either quadripolar or octopolar electrodes). Choosing the particular electrode entails deciding how many segments of the spinal cord are to be covered, with larger spacing allowing broader coverage. Alternatively, closer spacing allows better steering and electric field shaping. Additionally, multiple parallel electrodes and different configuration matrices can be constructed which can create extremely focused electrical fields.

\section{Figure 2}

The above graphic on the left shows a patient who had an L4,5 and L5,S1 anterior posterior fusion for back and leg pain from lumbar spondylolisthesis. She continued to have

both back and leg pain despite a successful lumbar reconstruction. An ANS (Advanced Neuromodulation System) tripole electrode was implanted in the thoracic spine. There are three columns of electrodes. The middle covers the back and the lateral columns provide stimulation into the legs. The electrode was placed in the operating room with fluoroscopy and intra-operative EMG monitoring under general anesthesia. 


\section{Plate electrodes}

Plate-type electrodes (or ribbon electrodes, paddle electrodes, or laminotomy electrodes) require a surgical procedure, laminotomy, and implantation under direct vision. Implantation under direct vision may be safer in the upper thoracic and cervical areas, where there is a risk of damaging the spinal cord with the large bore Touhy needle. Most implants can be done through a skin incision between 2.5 $\mathrm{cm}$ and $4 \mathrm{~cm}$ long, depending on the size of the patient and spinal anatomy. The amount of bony removal is usually minimal.

The main advantage of plate electrodes is their greater inherent stability, with less to migrate. Plate electrodes are also more energy efficient. Multiple arrays or different electrode configurations can be constructed with plate electrodes. As with percutaneous leads, there are varying lengths, and shapes-such as curved leads and hinged leads, all designed to help facilitate insertion and tailor the electrode selection to the patient. They are a preferred option in the case of previous spine surgery at the implant levels.

North et al. have published on comparison between plate and percutaneous electrodes. ${ }^{68}$ Laminectomy electrode placement, although more invasive than percutaneous placement, yielded significantly better clinical results in patients with failed back surgery syndrome at up to 3-year follow up. Clinical success was defined as at least $50 \%$ pain relief and patient satisfaction with treatment. Secondary outcome measures were ability to perform various activities of daily living, neurological function, and analgesic use. There is some theoretical evidence that shaping of the electrical field is possible with even more complex electrode arrays. Holsheimer et al. ${ }^{69}$ concluded that the transverse tripolar system enabled finer control of paresthesia. Electrical field steering could change the paresthesia area completely. Using transverse tripolar configurations increases the threshold for stimulation of dorsal roots. This results in a wider therapeutic range, wider paresthesia coverage, and a greater probability to fully cover the painful area with paresthesia.

\section{Rechargeable and Non-rechargeable Pulse Generators and Radio- Frequency Receivers}

Electrical stimulation consists of rectangular pulses delivered to the epidural space through implanted electrode via a power source. Two basic types of systems are currently available: an Internal Pulse Generator (IPG; also called the battery) or a radiofrequency (RF) coupled pulse generator with an implantable receiver. The later has largely fallen out of favor due to the inconveniences of having an external power source. However, the advent of the totally implantable, rechargeable pulse generator has surmounted the power requirement issues, which were previously the main RF advantage.

The totally implantable pulse generator contains a lithium battery. Activation and control occur through an external transcutaneous telemetry device. The IPG can be turned on and off through a small controller which the patient can carry. The controller also allows some control over the stimulation parameters. More extensive control of the unit can be achieved through a small portable unit which can be programmed by the physician. Life span of the battery varies with usage and with the utilized parameters (voltage, rate, pulse width, etc.). Most patients can expect that the battery will last from 2.5 to 4.5 years. Available lithium powered pulse generators allow stimulation with fine control of stimulation amplitude, pulse width and rate. Replacement of the battery requires a surgical procedure which is usually performed on an outpatient basis.

Radio frequency (RF) driven systems, consist of a passive receiver, implanted subcutaneously, and a transmitter which is worn externally. An antenna applied to the skin in correspondence of the receiver is connected to the transmitter, which sends the stimulation signals transcutaneously. RF systems have the inconvenience of having to wear the antenna and the radio-receiver, replacing the batteries on a regular basis, and ensuring proper contact of the antenna on the skin. These issues may be critical for individuals with limited upper-limb motor function. Other patients, particularly individuals who have reflex sympathetic dystrophy (RSD), may not tolerate the antenna taped to the skin. However, what one loses in convenience, however, is gained in power and flexibility. Currently only RF systems can provide a stimulation rate up to 1,400 $\mathrm{Hz}$. This might be beneficial in some patients with neuropathic chronic pain syndromes ${ }^{70}$ as well as in patients with extrapyramidal motor disorders.

\section{Complications}

With the proper expertise, permanent complications are rare. ${ }^{71}$ The most serious complications are paralysis or other severe neurological deficits, which are risks inherent in any spine surgery. Infection of the implanted hardware has occurred with a $3-5 \%$ rate. Persistent pain at the implant site has been seen in about $5 \%$ of patients. Recalcitrant cerebrospinal fluid leakage has been encountered in a few patients, requiring multiple surgical revisions. Breakage or malfunction of the implanted hardware, particularly the electrodes and the subcutaneous extension cables has been encountered in about $10 \%$ of the implanted systems. Painful stimulation, necessitating either repositioning or removal of the electrode, has also been reported in a number of cases.

\section{Conclusions}

The treatment of chronic pain remains challenging. Spinal cord stimulation has been performed for over 30 years, and slow but steady progress with this technology has been made. As the equipment and stimulation parameters are improved, selection criteria have been better defined and are slowly being expanded. More importantly, experience in the technique and the equipment has made SCS a much more reliable and safe modality. Like all the modalities performed for chronic pain management, its results are favorable. It is important to remember that the goal of neurostimulation is to reduce pain, rather than to eliminate pain. It has been shown to have a $50 \%$ improvement in pain relief, and reduce the use of more medications Very few other invasive modalities can claim this success rate with a few years of follow-up.

Careful follow-up of the patients is necessary for successful long-term satisfaction. Equipment related problems can arise at any time after implantation, such as discomfort at the pulse generator/radio receiver site, electrode breakage or migration, infection, etc., and an open dialogue with the patients is vital for the continuing successful implementation of the modality. Spinal cord stimulation has earned a well established and firm role in contemporary chronic pain management.

\section{References}

1. Waltz, J.M., Computerized percutaneous multi-level spinal cord stimulation in motor disorders. Appl Neurophysiol, 1982. 45(1-2): p. 73-92.

2. Melzack, R. and P.D. Wall, Pain mechanisms: a new theory. Science, 1965. 150(699): p. 971-9.

3. Shealy, C.N., J.T. Mortimer, and J.B. Reswick, Electrical inhibition of pain by stimulation of the dorsal columns: preliminary clinical report. Anesth Analg, 1967. 46(4): p. 489-91.

4. Hoppenstein, R., Electrical stimulation of the ventral and dorsal columns of the spinal cord for relief of chronic intractable pain: preliminary report. Surg Neurol, 1975. 4(1): p. 187-94. 
5. Larson, S.J., et al., A comparison between anterior and posterior spinal implant systems. Surg Neurol, 1975. 4(1): p. 180-6.

6. Lazorthes, Y., J.C. Verdie, and L. Arbus, [Anterior and posterior medullary analgesic stimulation, using a percutaneous implantation technic]. Acta Neurochir (Wien), 1978. 40(3-4): p. 277-83.

7. Dooley, D. Percutaneous electrical stimulation of the spinal cord. in Assoc Neurol Surg. 1975. Bal Harbour, FL.

8. Coburn, B., Electrical stimulation of the spinal cord: twodimensional finite element analysis with particular reference to epidural electrodes. Med Biol Eng Comput, 1980. 18(5): p. 573-84.

9. Coburn, B., A theoretical study of epidural electrical stimulation of the spinal cord--Part II: Effects on long myelinated fibers. IEEE Trans Biomed Eng, 1985. 32(11): p. 978-86.

10. Coburn, B. and W.K. Sin, A theoretical study of epidural electrical stimulation of the spinal cord--Part I: Finite element analysis of stimulus fields. IEEE Trans Biomed Eng, 1985. 32(11): p. 971-7.

11. Holsheimer, J., et al., Significance of the spinal cord position in spinal cord stimulation. Acta Neurochir Suppl, 1995. 64: p. 119-24.

12. Holsheimer, J. and J.J. Struijk, How do geometric factors influence epidural spinal cord stimulation? A quantitative analysis by computer modeling. Stereotact Funct Neurosurg, 1991. 56(4): p. 234-49.

13. Holsheimer, J. and W.A. Wesselink, Effect of anode-cathode configuration on paresthesia coverage in spinal cord stimulation. Neurosurgery, 1997. 41(3): p. 654-9; discussion 659-60.

14. Foreman, R.D., et al., Effects of dorsal column stimulation on primate spinothalamic tract neurons. J Neurophysiol, 1976. 39(3): p. 534-46.

15. Linderoth, B., et al., Dorsal column stimulation induces release of serotonin and substance $\mathrm{P}$ in the cat dorsal horn Neurosurgery, 1992. 31(2): p. 289-96; discussion 296-7.

16. Barolat, G., R. Schwartzman, and R. Woo, Epidural spinal cord stimulation in the management of reflex sympathetic dystrophy. Stereotact Funct Neurosurg, 1989. 53(1): p. 29-39.

17. Kumar, K., R.K. Nath, and C. Toth, Spinal cord stimulation is effective in the management of reflex sympathetic dystrophy. Neurosurgery, 1997. 40(3): p. 503-8; discussion 508-9.

18. Kemler, M.A., et al., Electrical spinal cord stimulation in reflex sympathetic dystrophy: retrospective analysis of 23 patients. J Neurosurg, 1999. 90(1 Suppl): p. 79-83.

19. Kemler, M.A., et al., Spinal cord stimulation in patients with chronic reflex sympathetic dystrophy. N Engl J Med, 2000. 343(9): p. 618-24

20. Kemler, M.A., et al., Spinal cord stimulation for chronic reflex sympathetic dystrophy--five-year follow-up. N Engl J Med, 2006. 354(22): p. 2394-6

21. Oakley, J. and R. Weiner, Spinal cord stimulation for complex regional pain syndrome: a prospective study of 19 patients at two centers. Neuromodulation, 1999. 2: p. 47-50.

22. Calvillo, O., et al., Neuroaugmentation in the treatment of complex regional pain syndrome of the upper extremity. Acta Orthop Belg, 1998. 64(1): p. 57-63.

23. Ebel, H., et al., Augmentative treatment of chronic deafferentation pain syndromes after peripheral nerve lesions Minim Invasive Neurosurg, 2000. 43(1): p. 44-50.

24. Cameron, T., Safety and efficacy of spinal cord stimulation for the treatment of chronic pain: a 20 -year literature review. J Neurosurg, 2004. 100(3 Suppl Spine): p. 254-67.

25. Law, J.D., Targeting a spinal stimulator to treat the 'failed back surgery syndrome’. Appl Neurophysiol, 1987. 50(1-6): p. 437-8.

26. Law, J.D., Clinical and technical results from spinal stimulation for chronic pain of diverse pathophysiologies. Stereotactic \& Functional Neurosurgery, 1992. 59(1-4): p. 21-4.

27. North, R.B., et al., Spinal cord stimulation electrode design A prospective randomized, controlled trial comparing percutaneous and laminectomy electrodes. Stereotact Funct Neurosurg, 1999. 73(1-4): p. 134.
28. Sharan, A., et al. Selective Dorsal Column Activation With Three Column Electrode Arrays Using Percutaneous and Paddle Leads. in 2007 AANS Annual Meeting. 2007. Washington, DC

29. Marchand, S., et al., The effects of dorsal column stimulation on measures of clinical and experimental pain in man. Pain 1991. 45(3): p. 249-57.

30. 5-year follow-up after spinal cord stimulator implantation. Neurosurgery, 1991. 28(5): p. 692-9.

31. Turner, J.A., J.D. Loeser, and K.G. Bell, Spinal cord stimulation for chronic low back pain: a systematic literature synthesis. Neurosurgery, 1995. 37(6): p. 1088-95; discussion 1095-6.

32. Burchiel, K.J., et al., Prospective, multicenter study of spina cord stimulation for relief of chronic back and extremity pain. Spine, 1996. 21(23): p. 2786-94.

33. North, R.B., D.H. Kidd, and S. Piantadosi, Spinal cord stim ulation versus reoperation for failed back surgery syndrome: a prospective, randomized study design. Acta Neurochir Suppl, 1995. 64: p. 106-8.

34. Augustinsson, L.E., Spinal cord electrical stimulation in severe angina pectoris: surgical technique, intraoperative physiology, complications, and side effects. Pacing Clin Electrophysiol, 1989. 12(4 Pt 2): p. 693-4.

35. de Jongste, M.J., et al., Effects of spinal cord stimulation on myocardial ischaemia during daily life in patients with severe coronary artery disease. A prospective ambulatory electrocardiographic study. Br Heart J, 1994. 71(5): p. 413-8.

36. de Jongste, M.J., et al., Efficacy of spinal cord stimulation as adjuvant therapy for intractable angina pectoris: a prospective, randomized clinical study. Working Group on Neurocardiology. J Am Coll Cardiol, 1994. 23(7): p. 1592-7.

37. Mannheimer, C., et al., Epidural spinal electrical stimulation in severe angina pectoris. Br Heart J, 1988. 59(1): p. 56-61.

38. Sanderson, J.E., et al., Epidural spinal electrical stimulation for severe angina: a study of its effects on symptoms, exercise tolerance and degree of ischaemia. Eur Heart J, 1992. 13(5): p. 628-33.

39. Hautvast, R.W., et al., Effect of spinal cord stimulation on myocardial blood flow assessed by positron emission tomography in patients with refractory angina pectoris. Am J Cardiol, 1996. 77(7): p. 462-7.

40. Vulink, N., et al., The effects of spinal cord stimulation on quality of life in patients with therapeutically chronic refrac tory angina pectoris. Neuromodulation, 1999: p. 33-40.

41. Hautvast, R.W., et al., Spinal cord stimulation in chronic intractable angina pectoris: a randomized, controlled efficacy study. Am Heart J, 1998. 136(6): p. 1114-20.

42. Mannheimer, C., et al., Electrical stimulation versus coronary artery bypass surgery in severe angina pectoris: the ESBY study. Circulation, 1998. 97(12): p. 1157-63.

43. Ekre, O., et al., Long-term effects of spinal cord stimulation and coronary artery bypass grafting on quality of life and survival in the ESBY study. Eur Heart J, 2002. 23(24): p. 1938-45.

44. Di Pede, F., et al., Immediate and long-term clinical outcome after spinal cord stimulation for refractory stable angina pectoris. Am J Cardiol, 2003. 91(8): p. 951-5.

45. Andersen, C., Complications in spinal cord stimulation for treatment of angina pectoris. Differences in unipolar and multipolar percutaneous inserted electrodes. Acta Cardiol, 1997. 52(4): p. 325-33.

46. Bagger, J.P., B.S. Jensen, and G. Johannsen, Long-term outcome of spinal cord electrical stimulation in patients with refractory chest pain. Clin Cardiol, 1998. 21(4): p. 286-8.

47. Eliasson, T., et al., Safety aspects of spinal cord stimulation in severe angina pectoris. Coron Artery Dis, 1994. 5(10): p. 845-50.

48. Sanderson, J.E., et al., Spinal electrical stimulation for intractable angina--long-term clinical outcome and safety. Eur Heart J, 1994. 15(6): p. 810-4.

49. Andersen, C., P. Hole, and H. Oxhoj, Does pain relief with spinal cord stimulation for angina conceal myocardial infarction? Br Heart J, 1994. 71(5): p. 419-21.
50. Murray, S., et al., Spinal cord stimulation significantly decreases the need for acute hospital admission for chest pain in patients with refractory angina pectoris. Heart, 1999 82(1): p. 89-92.

51. Gonzalez-Darder, J., V. Gonzalez-Martinez, and P. CanelaMoya, Cervical Spinal Cord Stimulation in the Treatment of Severe Angina Pectoris. Neurosurgical Quarterly, 1998. 8: p. 16-23.Augustinsson, L.E., T. Eliasson, and C. Mannheimer, Spinal cord stimulation in severe angina pectoris. Stereotactic \& Functional Neurosurgery, 1995 65(1-4): p. 136-41

52. Andersen, C., Does heart rate variability change in angin pectoris patients treated with spinal cord stimulation? Cardiology, 1998. 89(1): p. 14-8.

53. Hautvast, R.W., et al., Effect of spinal cord stimulation on heart rate variability and myocardial ischemia in patients with chronic intractable angina pectoris--a prospective ambulatory electrocardiographic study. Clin Cardiol, 1998. 21(1): p. 33-8.

54. Meller, S.T. and G.F. Gebhart, A critical review of the afferent pathways and the potential chemical mediators involved in cardiac pain. Neuroscience, 1992. 48(3): p. 501-24.

55. Thamer, V., et al., Pain and myocardial ischemia: the role of sympathetic activation. Basic Res Cardiol, 1990. 85 Suppl 1: p. 253-66.

56. Norrsell, H., et al., Effects of spinal cord stimulation and coronary artery bypass grafting on myocardial ischemia and heart rate variability: further results from the ESBY study. Cardiology, 2000. 94(1): p. 12-8.

57. Eddicks, S., et al., Thoracic spinal cord stimulation improve functional status and relieves symptoms in patients with refractory angina pectoris: the first placebo-controlled randomised study. Heart, 2007. 93(5): p. 585-90.

58. Hirshberg, R.M., et al., Is there a pathway in the posterior funic ulus that signals visceral pain? Pain, 1996. 67(2-3): p. 291-305.

59. Ceballos, A., et al., Spinal cord stimulation: a possible therapeutic alternative for chronic mesenteric ischaemia. Pain, 2000. 87(1): p. 99-101.

60. Krames, E. and D. Mousad, Spinal cord stimulation reverses pain and diarrheal episodes of irritable bowel syndrome: a case report. Neuromodulation, 2004. 7: p. 82-88.

61. Khan, Y., S. Raza, and E. Khan, Application of spinal cord stimulation for the treatment of abdominal visceral pain syndromes: case reports. Neuromodulation, 2005. 8: p. 14-27.

62. Tiede, J.M., et al., The use of spinal cord stimulation in refractory abdominal visceral pain: case reports and literature review. Pain Pract, 2006. 6(3): p. 197-202.

63. Kapur, S., H. Mutagi, and J. Raphael, Spinal cord stimulation for relief of abdominal pain in two patients with familial Mediterranean fever. Br J Anaesth, 2006. 97(6): p. 866-8.

64. Kapural, L., et al., Spinal cord stimulation is an effective treatment for the chronic intractable visceral pelvic pain. Pain Med, 2006. 7(5): p. 440-3.

65. Ness, T.J. and G.F. Gebhart, Visceral pain: a review of experimental studies. Pain, 1990. 41(2): p. 167-234.

66. Mitchell, G., Anatomy of the autonomic nervous system. 1953, Edinburgh: E \& S Livingstone.

67. North, R.B., et al., Spinal cord stimulation electrode design: a prospective, randomized, controlled trial comparing percutaneous with laminectomy electrodes: part II-clinical outcomes. Neurosurgery, 2005. 57(5): p. 990-6; discussion 990-6.

68. Holsheimer, J., et al., Clinical evaluation of paresthesia steering with a new system for spinal cord stimulation. Neurosurgery, 1998. 42(3): p. 541-7; discussion 547-9.

69. Alo, K., et al. The treatment of refractory reflex sympathetic dystrophy with higher frequency tripolar dual octrode spinal cord stimulation: a case report. . in Abstracts of the 4th International Congress of the INS. 1998.

70. Barolat, G., W. Peacock, and L. Staudt, Pain and spasticity, in Spine Surgery: Techniques, Complications Avoidance and Management, E. Benzel, Editor. 1999, Churchill Livingstone. p. 863-876. 